\title{
Antenatal screening for intrauterine growth retardation with umbilical artery Doppler ultrasonography
}

\author{
R B Beattie, J C Dornan
}

\begin{abstract}
To assess the usefulness of continuous wave Doppler ultrasonography as an antenatal screening tool for the detection of intrauterine growth retardation and fetal compromise 2097 singleton pregnancies were studied. Umbilical artery velocity waveforms were obtained at 28,34 , and 38 weeks of gestation, from which the pulsatility index, $\mathrm{A} / \mathrm{B}$ ratio, and resistance parameter were calculated.

No abnormal features or indices of neonatal outcome were adequately predicted. The most sensitive index for being delivered of a growth retarded infant ( $<5$ th centile birth weight for gestation) was an $A / B$ ratio at 34 weeks (sensitivity $40 \%$, specificity $84 \%$ ). Other measures that show poor neonatal nutritional state (ponderal index, skinfold thickness, and ratio of mid-arm circumference to head circumference) were even less well predicted. Acute and chronic hypoxia as determined by Apgar score, $\mathrm{pH}$ in blood from the cord artery, and packed cell volume correlated poorly with umbilical artery waveform indices, and there was no obvious difference between the indices of those who subsequently required operative or instrumental delivery for fetal distress and those requiring no intervention. There were three unexplained stillbirths in the series, in each of which the fetus had shown waveform patterns that suggested increased peripheral resistance, though the technique did not appear to be useful for predicting the time of subsequent death.

Screening for small for dates babies in a three stage programme was of no value regardless of the threshold or index chosen. Obstetricians should resist the temptation to introduce screening with Doppler ultrasonography until its proper role has been determined.
\end{abstract}

\section{Introduction}

Doppler ultrasonography has been used in obstetrics for the real time non-invasive direct measurement of the shape of the velocity waveform in the uteroplacental, fetoplacental, and fetal circulations. The association of a reduced diastolic component of the umbilical artery waveform with intrauterine fetal growth retardation has been suggested. Trudinger $e t a l$ and Reuwer $e t$ al found that fetuses identified clinically and biophysically as being growth retarded invariably showed reduction, absence, or reversal of the diastolic waveform component, which was associated with a poor perinatal outcome. ${ }^{1.3}$

We designed a study to assess the feasibility of using continuous wave Doppler ultrasonography of the umbilical artery as an antenatal screening tool for the detection of intrauterine fetal growth retardation and fetal compromise. Various indices have been used to describe the shape of the waveform in the umbilical artery, ${ }^{+-6}$ and the three most commonly used are shown in figure 1. These dimensionless indices describe the shape of the waveform and reflect the distal vascular impedance. All three are independent of the angle of insonation but only the pulsatility index can be used to describe waveforms with "zero diastolic" or reversed diastolic flow. As diastolic flow reduces, the resistance parameter approaches unity or a negative value and the $\mathrm{A} / \mathrm{B}$ ratio approaches infinity. The pulsatility index varies from cycle to cycle owing to minor differences in heart rate ${ }^{7}$ and therefore an average from more than one cycle should be obtained. When diastolic waveforms are low small changes in the measured diastolic component will have a pronounced effect on the final ratio and therefore large.values of all indices are inaccurate.

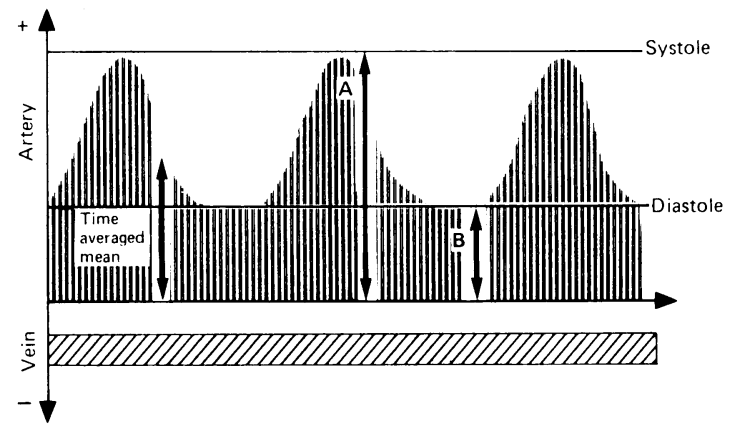

FIG $1-$ Calculation of waveform indices in Doppler ultrasonography. $P$ ulsatility index $=A-B /$ time averaged mean $; A / B$ ratio $=A / B$; resistance parameter $=A-B / A$

\section{Patients and methods}

In all, 2097 ultrasonically dated singleton pregnancies were entered into the study between 1 August 1986 and 31 July 1987 and were examined at 28, 34, and 38 weeks' gestation, as is the pattern for "shared care" patients attending our unit. The only selection criteria applied were that they should be singleton pregnancies and that mothers should have attended our hospital within seven days of their 28th gestational week. This provided a sample of $62 \%$ of the 3400 singleton pregnancies for the year, and excluded private patients, late bookings, those with altered dates who attended after 29 weeks, and late referrals from other hospitals. The attendance rate of the undelivered patients was $95 \%$ and $99 \%$ at 34 and 38 weeks' gestation, respectively.

There was no statistically significant difference between the women who were screened and those with singleton pregnancies who were not screened in the known determinants of fetal outcome such as age, parity, social class, and smoking habit. The mean age of women in the study group was 26.3 (SD 5.5) years and mean parity $1.3(\mathrm{SD} 1.4) ; 256(12 \%)$ required caesarean sections and $170(8 \%)$ required instrumental or operative intervention for fetal distress.
Correspondence to: Dr Br.Med f 1989:298:631-5 
Ethical approval for the study was granted by the Queen's University of Belfast ethics committee. All patients with confirmed singleton pregnancies were first approached by their midwives or obstetricians at the booking visit. The outline of the study was explained verbally and a written note of explanation given. They were invited to join the study, and if they consented their antenatal chart was marked for ease of identification. Further explanation of the study and basic principles of Doppler scanning were given verbally by the ultrasonographers at the first scan and informed verbal consent thus obtained. The main reasons for refusal to enter the study were concerns about further delay while attending the antenatal clinic and distrust of the safety of a new technique for fetal monitoring.

A Doptek 9000 spectrum analyser (Doptek Limited, England) was used for measuring the waveforms in the umbilical artery. The frequency of the wall vessel filter was $200 \mathrm{~Hz}$. The sonograms were obtained from the periumbilical region of the mother's abdomen and waveform pattern recognition was used to identify the umbilical cord. The mothers were examined while lying in a semirecumbent position, having rested in a non-smoking waiting room for at least 15 minutes before examination. To reduce distortion of the Doppler sonogram a sample of approximately 10 cycles was obtained during fetal apnoea and in the absence of any audible or visible artefacts due to fetal activity. A successful Doppler examination was achieved within an average of five minutes. A light pen was used to mark the length, systolic peak, and end diastolic components of the three best cycles. These were selected on the basis of absence of high intensity areas near the baseline due to vessel wall movements; a concentration of high intensity outline near the maximum frequency, which indicates insonation of the centre of the vessel lumen; and an absence of high frequency "noise," which causes inaccurate computer tracing of $7 / 8$ of the maximum frequency envelope that is used to calculate the pulsatility index.

The pulsatility index, $\mathrm{A} / \mathrm{B}$ ratio, resistance parameter, and duration of the cycle were all automatically calculated and averaged for each study. We defined a value $>90$ th centile at each gestational age as abnormal as this would produce the largest possible group that could effectively be followed up in a screening study and because the frequency distribution of the waveform indices was unknown. The results were stored on a microcomputer with an integral hard disc. A hard copy of each trace was also produced.

The results were not seen by the clinicians and all measurements were made by one of three observers: a research midwife, a medical technician, and an obstetric research fellow. A concurrent reproducibility study showed that the interobserver and intraobserver errors were non-significant (Kruskal-Wallis test, $\mathrm{p}=$ 0.65 interobserver, $\mathrm{p}=0.34$ intraobserver for pulsatility index).

As well as the traditional measures of perinatal outcome (birth weight for gestational age, Apgar score, crown-heel length, and occipitofrontal head circumference) a number of additional variables were recorded. To identify fetuses with poor intrauterine nutrition the ponderal index, ${ }^{x}$ ratio of mid-arm circumference to head circumference, ${ }^{4}$ and skinfold thickness ${ }^{10}$ were recorded. Acidosis at birth was assessed from $\mathrm{pH}$ in blood from the umbilical artery, and the packed cell volume of the fetal blood was measured, as chronic hypoxia is associated with polycythaemia."

At delivery the midwife or obstetrician took a blood sample from the umbilical artery after double clamping the cord and measured the $\mathrm{pH}$ on a blood gas analyser (Instrumentation Laboratory 1312); the packed cell volume was determined using a standard centrifuge.
On the day after delivery skinfold measurements were taken as the average of two readings from the left subscapular sites and triceps with a pair of Harpenden skinfold calipers, which can be read to $0.1 \mathrm{~mm}$. These were left on the skin for 30 seconds or until the reading had stabilised. The mid-arm circumference was taken as the average of two measurements from the left side of the body, measured to the nearest $3 \mathrm{~mm}$.

The details of the course and outcome of the pregnancy were entered into a data file on the microcomputer that contained no information about Doppler studies on the patients; this file was linked to the results of Doppler studies by the antenatal number of each patient. The files were combined and analysed with the statistical package for the social sciences, extended version (SPSS X).

\section{Results}

The frequency distributions of the three Doppler indices were positively skewed at 28,34 , and 38 weeks, and this was not corrected by logarithmic transformation. At 28 weeks the pulsatility index showed a bimodal distribution with the smaller mode comprising about $15 \%$ of all cases. ${ }^{12}$ This pattern was not found at 34 and 38 weeks. As expected all three indices of distal vascular impedence reduced with increasing gestational age, implying falling placental impedance, ${ }^{13}$ although there were six cases with a zero diastolic waveform component.

In view of the frequency distribution and the influence of gestational age, graphs for pulsatility index, $\mathrm{A} / \mathrm{B}$ ratio, and resistance parameter at specific ages were constructed. A value $>90$ th centile for gestational age was defined as abnormal, and parametric range definitions were derived for comparison with other studies (table I).

TABLE I-Reference ranges for umbilical artery Doppler ultrasonography

\begin{tabular}{|c|c|c|c|c|c|}
\hline & \multirow[b]{2}{*}{$\begin{array}{l}\text { Weeks' } \\
\text { gestation }\end{array}$} & \multicolumn{4}{|c|}{ Values obtained } \\
\hline & & $\begin{array}{l}\text { At 10th } \\
\text { centile }\end{array}$ & $\begin{array}{l}\text { At 90th } \\
\text { centile }\end{array}$ & $\begin{array}{c}\text { Mean } \\
-2 S D\end{array}$ & $\begin{array}{l}\text { Mean } \\
+2 \mathrm{SD}\end{array}$ \\
\hline Pulsatility index & $\left\{\begin{array}{l}28 \\
34 \\
38\end{array}\right.$ & $\begin{array}{l}0.96 \\
0.82 \\
0.75\end{array}$ & $\begin{array}{l}1.73 \\
1.28 \\
1.17\end{array}$ & $\begin{array}{l}0.66 \\
0.60 \\
0.56\end{array}$ & $\begin{array}{l}1.84 \\
1.52 \\
1.35\end{array}$ \\
\hline $\mathrm{A} / \mathrm{B}$ ratio & $\left\{\begin{array}{l}28 \\
34 \\
38\end{array}\right.$ & $\begin{array}{l}2.43 \\
2.09 \\
1.91\end{array}$ & $\begin{array}{l}4 \cdot 29 \\
3 \cdot 44 \\
3 \cdot 00\end{array}$ & $\begin{array}{l}1.60 \\
1.40 \\
1.41\end{array}$ & $\begin{array}{l}5 \cdot 05 \\
4 \cdot 08 \\
3 \cdot 40\end{array}$ \\
\hline Resistance parameter & $\left\{\begin{array}{l}28 \\
34 \\
38\end{array}\right.$ & $\begin{array}{l}0.58 \\
0.52 \\
0.47\end{array}$ & $\begin{array}{l}0 \cdot 76 \\
0 \cdot 70 \\
0 \cdot 66\end{array}$ & $\begin{array}{l}0.54 \\
0 \cdot 46 \\
0 \cdot 42\end{array}$ & $\begin{array}{l}0.81 \\
0.76 \\
0.71\end{array}$ \\
\hline
\end{tabular}

\section{INTRAUTERINE FETAL GROWTH RETARDATION}

The sensitivity, specificity, and positive and negative predictive values of the pulsatility index, A/B ratio, and resistance parameter in identifying the fetuses with a subsequent birth weight $<5$ th centile for gestational age were determined (table II). All three indices were significantly related to the birth weight of the fetus in relation to the 5 th centile for gestational age $(\mathrm{p}<0.05)$. The most sensitive index was the $\mathrm{A} / \mathrm{B}$ ratio, although a sensitivity of only $31 \%$ was achieved at 28 weeks and $40 \%$ at 34 weeks. When any of the three results were abnormal the sensitivity remained low (43\%); $17 \%$ to $19 \%$ of the population was identified as being at risk.

Curves for receiver operating characteristics were produced for all waveform indices at all three gestational ages. Figure 2 shows the relation between the sensitivity and specificity of the best single index, the $\mathrm{A} / \mathrm{B}$ ratio at 34 weeks. Selecting threshold values lower than the 90th centile to define an abnormal waveform index resulted in improved sensitivity but at considerable loss of specificity. 
TABLE II - Screening for intrauterine fetal growth retardation with Doppler ultrasonography

\begin{tabular}{|c|c|c|c|c|c|c|}
\hline & \multirow[b]{2}{*}{$\chi^{2}(\mathrm{df}=1)$} & \multirow[b]{2}{*}{$\mathrm{p}$ Value } & \multirow[b]{2}{*}{ Sensitivity } & \multirow[b]{2}{*}{ Specificity } & \multicolumn{2}{|c|}{ Predictive value } \\
\hline & & & & & Positive & Negative \\
\hline \multicolumn{7}{|c|}{28 weeks' gestation ${ }^{\star}$} \\
\hline Pulsatility index & $18 \cdot 1$ & $\mathrm{p}<0.0001$ & 28 & 89 & 11 & 97 \\
\hline $\mathrm{A} / \mathrm{B}$ ratio & $26 \cdot 5$ & $\mathrm{p}<0.0001$ & 31 & 90 & 12 & 97 \\
\hline Resistance parameter & $5 \cdot 4$ & $\mathrm{p}<0.0198$ & 14 & 94 & 9 & 96 \\
\hline Any index & $32 \cdot 9$ & $\mathrm{p}<0.0001$ & 43 & 84 & 11 & 97 \\
\hline \multicolumn{7}{|c|}{34 weeks' gestation $\dagger$} \\
\hline Pulsatility index & $23 \cdot 4$ & $\mathrm{p}<0.0001$ & 32 & 89 & 12 & 97 \\
\hline $\mathrm{A} / \mathrm{B}$ ratio & $23 \cdot 9$ & $\mathrm{p}<0.0001$ & 40 & 84 & 11 & 97 \\
\hline Resistance parameter & $24 \cdot 5$ & $\mathrm{p}<0.0001$ & 33 & 88 & 12 & 97 \\
\hline Any index & $22 \cdot 9$ & $\mathrm{p}<0.0001$ & 43 & 82 & 10 & 97 \\
\hline \multicolumn{7}{|c|}{38 weeks' gestation $\dagger$} \\
\hline Pulsatility index & $11 \cdot 5$ & $\mathrm{p}<0.0007$ & 31 & 86 & 9 & 97 \\
\hline A/B ratio & $14 \cdot 6$ & $\mathrm{p}<0.0001$ & 30 & 89 & 11 & 97 \\
\hline Resistance parameter & $8 \cdot 2$ & $\mathrm{p}<0.0042$ & 31 & 84 & 8 & 96 \\
\hline Any index & $14 \cdot 3$ & $\mathrm{p}<0.0002$ & 40 & 82 & 9 & 97 \\
\hline
\end{tabular}

*Use of any index identified $17 \%$ of the population as being at risk. tUse of any index identified $19 \%$ of the population as being at risk.

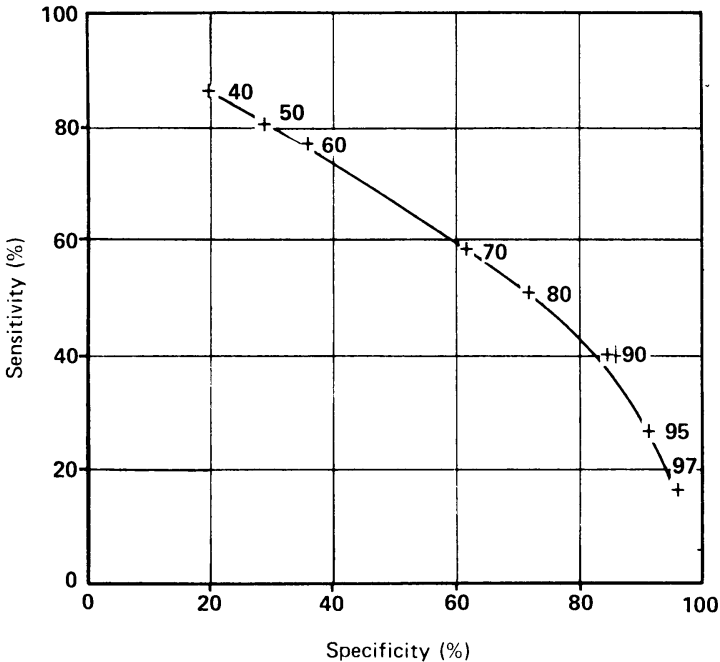

FIG $2-$ Sensitivity and specificity of $A / B$ ratio at different percentiles (40th to 97th) used as threshold to define abnormal waveforms

\section{FETAL NUTRITION}

A ponderal index (birth weight $(\mathrm{g}) \times 100 /$ crown-heel length $\left.(\mathrm{cm})^{3}\right)<2 \cdot 32$ has been defined as representing a malnourished neonate. ${ }^{14}$ In our series, the $\chi^{2}$ test failed to reach significance for any of the indices of nutrition. Scatterplots for ponderal index, ratio of mid-arm circumference to head circumference, and subscapular skinfold thickness showed the poor ability of umbilical artery waveform analysis to identify fetuses that subsequently showed morphometric evidence of intrauterine growth retardation. This is illustrated in figure 3 for the ponderal index. If the technique of umbilical artery Doppler velocimetry is to be of value for screening the fetuses with an abnormal outcome should have had pulsatility indices $>90$ th centile and those with normal outcome should have had waveform indices within the area representing normal neonatal outcome.

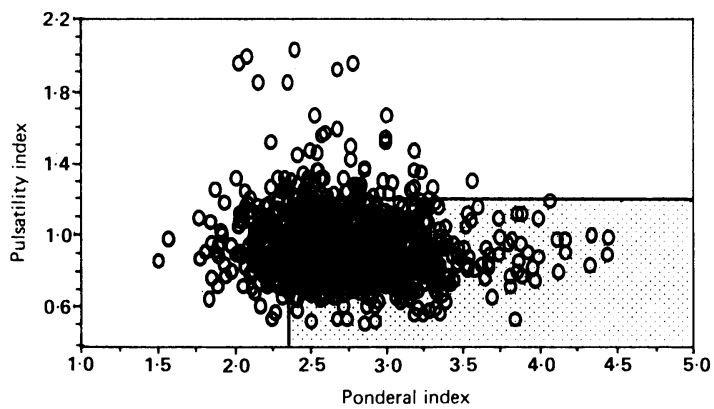

FIG 3-Pulsatility index at 38 weeks related to ponderal index of neonate. Ruled off area (bottom right) represents fetuses with normal neonatal outcome and Doppler index
The distribution shows the poor sensitivity and specificity of the technique. A similar pattern was found for the other indices of nutrition.

\section{FETAL HYPOXIA}

Chronic fetal hypoxia is associated with compensatory polycythaemia (packed cell volume $>0 \cdot 65$ ). Only four of the 13 babies affected had shown abnormal velocity waveforms (fig 4); the $\mathrm{A} / \mathrm{B}$ ratio at 38 weeks was the best index but was not significantly related to outcome ( $\chi^{2}$ test with Yates's correction).

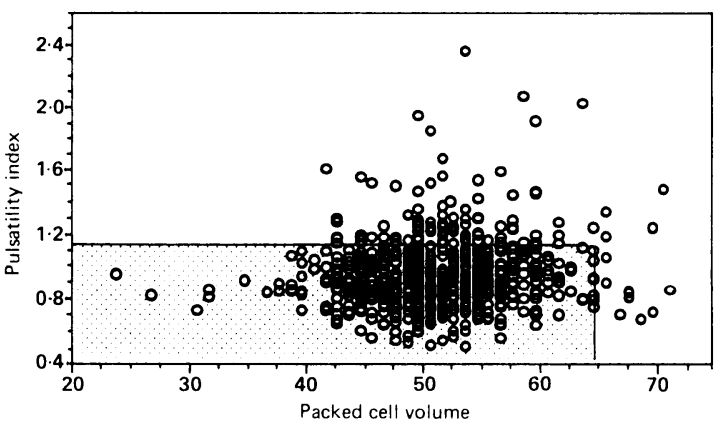

FIG 4-Pulsatility index at 38 weeks related to packed cell volume in blood taken from umbilical artery at birth. Ruled off area (bottom left) represents fetuses with normal packed cell volume and Doppler index

Thus the test is not discriminant for haemorrheological evidence of chronic hypoxia.

ACUTE FETAL HYPOXIA

Acute fetal hypoxia determined by the traditional Apgar scores and acidaemia at birth determined by $\mathrm{pH}$ of blood in the cord artery could not be usefully anticipated with Doppler analysis. When the $\chi^{2}$ test was applied to fetal acidaemia $(\mathrm{pH}<7 \cdot 1)$ and abnormal waveform indices, it was significant for any index only at 38 weeks $(\mathbf{p}<0 \cdot 05)$. An abnormal pulsatility index at 38 weeks identified only $31 \%$ of fetuses with subsequent acidaemia at birth.

\section{FETAL DISTRESS}

Fetal distress was diagnosed clinically in $170(8 \%)$ cases; in these instrumental or operative delivery was required. There was no significant difference in the occurrence of abnormal waveform indices between the group with fetal distress and the rest of the population, which required no intervention. The ranges and distributions of values for the pulsatility index at 38 weeks were almost identical in fetuses clinically diagnosed as distressed and those not.

\section{PERINATAL MORTALITY}

Thirteen babies died, giving a perinatal mortality rate of $6 \cdot 3$ per 1000 . Seven were neonatal deaths and six were stillbirths, and perinatal death was not adequately predictable on the basis of Doppler studies (table III)

TABLE III - Perinatal death related to results of Doppler ultrasound

\begin{tabular}{lcc} 
& Gestation & Doppler result \\
\hline Stillbirths $^{\star}$ Anencephaly & & \\
Placental abruption & 34 weeks & Normal \\
Placental abruption & 33 weeks & Normal \\
Unexplained & 38 weeks & Abnormal \\
Unexplained & 28 weeks & Abnormal \\
Unexplained & 33 weeks & Abnormal \\
Neonatal deathst: & 38 weeks & Abnormal \\
Neural tube defect & & \\
Multiple anomalies; small for dates & & Normal \\
Fallot's tetralogy & & Normal \\
Preterm; respiratory disease syndrome & & Abnormal \\
Preterm; hypoplastic lungs & & Normal \\
Placental abruption & & Abnormal \\
Rhesus hydrops & & Normal \\
& & Normal \\
\hline
\end{tabular}

$\star$ Mortality was $2 \cdot 9 / 1000$

tMortality was $3 \cdot 4 / 1000$ 
The association between major fetal anomalies and abnormal fetoplacental waveforms has been documented, and it is not surprising that the neonate in our study with a congenital cardiovascular defect (Fallot's tetralogy) had abnormal Doppler results before birth. In all three unexplained stillbirths abnormal waveforms were the only risk factor identified, although the time interval between the abnormal Doppler result and fetal death varied from six days to six weeks. About 200 other fetuses ( $10 \%$ of the study population) were also identified in this way as being at risk but had a normal outcome.

\section{ZERO DIASTOLIC COMPONENT}

With the Doptek 9000 analyser and a $200 \mathrm{~Hz}$ filter, six results showed an absent diastolic waveform component at 28 weeks. In three cases the waveform indices reverted to normal and the outcome of pregnancy was normal. One fetus was small for dates and two were unexplained stillbirths.

\section{Discussion}

In recent years there has been increasing interest in Doppler ultrasound as useful in obstetric practice for diagnosing intrauterine fetal growth retardation, perinatal asphyxia, and fetal distress. To provide useful, comparable results researchers must define cut off points for abnormal waveform indices based on the knowledge of the distribution of these variables in a large unselected population and the effect of advancing gestation. A fixed value cannot arbitrarily be selected from a study of a small population; indices may not be normally distributed, and this is the case for Doppler waveform indices in the umbilical artery. Hence, nonparametric statistics are appropriate for descriptive and comparative statistics in studies that use Doppler waveform analysis.

There has been some concern about the way online spectrum analysers calculate the pulsatility index and it cannot be assumed that normal range values can be generalised to different systems. For the Doptek 9000 spectrum analyser at least, umbilical artery Doppler indices should be graded, based on our centile values at 28,34 , and 38 weeks. Using the same model of spectrum analyser we observed an obstetrically normal population of 90 mothers at intervals of two weeks from 20 weeks until delivery in parallel with our main study. Analysis of these data showed that this series could be superimposed on the results from the study group, and thus it is reasonable to use our reference ranges for the study group to cover the period from 28 to 38 weeks (table I).

In screening for intrauterine fetal growth retardation as defined by birth weight $<5$ th centile for gestational age the technique described was not satisfactorily sensitive. A sensitivity of only $43 \%$ was achieved for any abnormal result ( $>90$ th centile) of any of the indices (pulsatility index, $\mathrm{A} / \mathrm{B}$ ratio, resistance parameter) at any of the gestational ages studied $(28,34$, and 38 weeks) to identify a fetus with intrauterine fetal growth retardation. This is unacceptably low for a condition affecting only $5 \%$ of fetuses if over half are not identified. Sensitivity improved when percentile thresholds for an abnormal waveform were lowered but specificity decreased appreciably; thus regardless of the cut off point or index chosen the technique is unsuitable for detection of intrauterine fetal growth retardation.

For intrauterine fetal growth retardation defined as birth weight $<10$ th centile and with the same three waveform indices, Mulders et al found the screening technique applied at 34 weeks' gestation to be of no more value in detecting growth retardation than physical examination combined with ultrasonography (sensitivity $53 \%$, specificity $88 \%$ for pulsatility index $<1 \cdot 1$ and $\mathrm{A} / \mathrm{B}$ ratio $<3 \cdot 0){ }^{7}$ Fleischer et al ${ }^{15}$ reported better results for the same definition of intrauterine fetal growth retardation when an abnormal $\mathrm{A} / \mathrm{B}$ ratio was used (sensitivity $78 \%$, specificity $83 \%$ ).

Intrauterine fetal growth retardation is, however, an imprecise multifactorial condition and does not necessarily imply diminished fetoplacental circulation; it lacks a universally accepted definition and may be difficult to detect even in the neonate; thus other indices of neonatal nutritional state have been developed. None of those used in this study (ponderal index, ratio of mid-arm circumference to head circumference, and skinfold thickness) correlated well with the abnormal Doppler results obtained, suggesting that the screening technique described cannot adequately detect fetal malnutrition.

At 28 weeks' gestation a subgroup of fetuses showed a basic difference in fetoplacental haemodynamics that was reflected in an elevated umbilical artery pulsatility index. This has not been recognised previously, and may be related to a transient phase of abnormality in placental development.

Blood viscosity, a determinant of flow, is governed mainly by the packed cell volume, deformability of erythrocytes, total serum concentration of protein, and plasma concentration of fibrinogen. ${ }^{16}$ In chronic fetal hypoxia there is increased fetal erythropoiesis ${ }^{11}$ with an increase in the larger immature erythrocytes, and thus high viscosity has been reported in the umbilical blood of acidotic neonates ${ }^{17}$ and in cases of fetal growth retardation..$^{18}$ Chronic fetal distress correlates well with reduced umbilical venous blood flow. ${ }^{19}$ If the umbilical artery Doppler technique could identify fetuses with chronic hypoxia, elevated waveform indices would have been associated with a high packed cell volume in blood from the umbilical artery; this association was not found.

The Apgar score has traditionally been used as a measure of fetal outcome, though the correlation between this subjective score and $\mathrm{pH}$ in the umbilical artery is poor ${ }^{20}$ and a low $\mathrm{pH}$ correlates better with the neurological condition of the neonate than does a low Apgar score. ${ }^{21}$ In this series we found a poor correlation between the last result of Doppler ultrasonography before delivery and fetal acidaemia at birth, defined as a cord $\mathrm{pH}<7 \cdot 1$, and there was no correlation with a 5 minute Apgar score $<7$.

A comparative study of the same three umbilical artery waveform indices at 34 weeks' gestation suggested that they may predict fetal distress better than intrauterine fetal growth retardation. ${ }^{7}$ Fetal distress is a complex diagnosis and is often based on a clinical decision aided by a fetal heart tracing taken during labour. In our study the range and distribution of the antenatal Doppler indices did not differ in fetuses who were subjected to operative or instrumental intervention for fetal distress compared with those not requiring any intervention. Trudinger et al suggested that the availability of umbilical artery waveform analysis is associated with improved obstetric decision making, reduced fetal distress, and improved neonatal outcome. ${ }^{3}$ When it is to be used in an unselected population, we cannot concur.

The low incidence of unexplained stillbirths in our study (3/2097) suggests that their association with waveform abnormalities should not be overemphasised; a much larger trial would be required to prove the association. These results, however, suggest that there may be a primary or secondary disturbance in fetoplacental impedance in at least some of the fetuses that are currently classified as unexplained stillbirths.

Whether the fetus or the placenta determines the fetoplacental blood flow has long been debated. Some 
workers have suggested a fetal role in the causation of abnormal umbilical artery waveforms in pregnancies complicated by major congenital abnormalities. Meizner et al studied umbilical and uterine artery waveforms, using continuous wave Doppler ultrasound to derive $\mathrm{A} / \mathrm{B}$ ratios, and found that 17 out of 32 cases of major congenital abnormalities had abnormal umbilical waveforms whereas only two had equivocal uterine waveforms, suggesting that a fetal mechanism was operative in over half the cases." Trudinger and Cook reported on 26 fetuses with major abnormalities and found that though half had abnormal umbilical artery waveforms all had normal uterine waveforms. They suggested that in the group with abnormal artery waveforms abnormal fetuses had triggered obliteration of small arteries in the placenta. Rochelson and colleagues speculated that when uterine artery patterns are abnormal, suggesting reduced perfusion, there may be placental damage or failure of adequate villous development. ${ }^{24}$ Normal uterine and abnormal umbilical waveforms may be due to failure of placental angiogenesis or an acute vasospasm or thrombosis.

The pathological change that occurs in the umbilical artery waveform, thought to be the result of increased vascular impedance distal to the vessel, is a progressive reduction in the diastolic component of the waveform; in some cases it may be absent or reversed such that fetoplacental flow seems to occur only during systole. It should, however, be remembered that the popular terminology of "absent" or "zero diastolic" flow does not imply absence of flow in diastole but rather a forward or reverse flow with a frequency shifted velocity waveform of less than the frequency of the vessel wall filter for that system. It is therefore mandatory that this frequency is specified in all publications relating to these terms.

An absent diastolic waveform component from the umbilical artery has been associated with altered left ventricular function in cases of intrauterine growth retardation and multiple congenital abnormalities with high perinatal morbidity and a perinatal mortality rate of $40-42 \% .{ }^{2+2}$ The loss of the normal forward diastolic component in the umbilical artery in late pregnancy has a multifactorial basis. In the trisomic fetus the histological appearance of the placenta and the associated sonogram is immature, comparable with that seen early in the second trimester. ${ }^{2+}$

Comparison with other studies is difficult as the frequency of the wall filter is often unspecified. Our results are broadly in keeping with other studies, which show a perinatal mortality of $33 \%$, and we agree that though absence of a diastolic component may precede obvious fetal growth retardation and may identify a fetus at risk, it does not aid in timing delivery and may in fact be associated with a completely normal outcome of pregnancy. Obstetric intervention solely on the basis of this finding is unjustified.

This study suggests that it is unhelpful to use umbilical artery Doppler ultrasonography to screen for poor fetal growth as measured in neonates by the traditional criteria as well as by ponderal index, ratio of mid-arm circumference to head circumference, and skinfold thickness. These may not be the most relevant features of outcome. Doppler ultrasonography of umbilical artery waveforms may be of clinical use as part of a wider biophysical profile of the fetus. It may be the answer - but to what question?

Until we more fully understand the physiological basis and relevance of these abnormal waveforms, the use of Doppler analysis of umbilical artery waveforms in clinical practice should be a tentative one. In our series only eight of 5730 Doppler studies had an absent diastolic component, a waveform pattern with very poor prognostic significance. Two of these were associated with subsequent unexplained stillbirths and one with a growth retarded fetus; the three other fetuses were appropriately grown and delivered in good condition.

Biophysical assessment of the fetus has allowed us to study fetal growth, fetal behaviour, and the fetal environment, and Doppler ultrasound is helping us study the normal and abnormal dynamic physiological state of the fetus. We concur with Neilson's caution that the proper role of Doppler ultrasonography has not yet been determined. ${ }^{26}$ Obstetricians should resist the temptation to introduce it prematurely as a panacea.

We thank the Northern Ireland Council for Orthopaedic Development for microcomputer equipment and software. We also thank $\mathrm{C}$ Clements, medical technician, and B McGrath, research midwife.

1 Trudinger BJ, Giles WB, Cook CM, Bombardieri J, Collins L. Fetal umbilical arterv flow waveforms and placental resistance: clinical significance. $\mathrm{Br} 7$ Obstet Gynaecol 1985:92:23-30.

2 Reuwer PJHM, Sijmons EA, Rietman GW, Van Tiel MWM, Bruinse HW Intrauterine growth retardation: prediction of perinatal distress by Doppler ultrasound.

3 Trudinger BJ, Giles WB, Cook CM, Connelly A, Thompson RS. Umbilical artery flow velocity waveforms in high-risk pregnancy. Lancet 1987:i: artery flow

+ Gosling RG, King DH. Continuous wave ultrasound as an alternative and complement to $x$-ravs in vascular examinations. In: Reneman RS, ed. Cardionascular applications of ultrasound. New York: Elsevier North Holland, 1974:266.

5 Gosling RG. Extraction of physiological information from spectrum analysed Doppler-shifted continuous wave ultrasound signal obtained non-invasively from the arterial tree. In: Hill DM, Watson BW, eds. IEE medical electronic monograph 21. Stevenage: Peter Peregrinus, 1976:73-125.

6 Pourcelot L. Applications cliniques de l'examen Doppler transcutane. In: Peroneau P, ed. l'elocimetric ultrasonore Doppler. Paris: INSERM, 1975 $213-40$.

7 Mulders LGM, Wijn PFF, Jongsma HW, Hein PR. A comparative study of three indices of umbilical blood flow in relation to prediction of growth

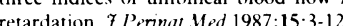

8 Lubchenco L.O, Bard $\mathrm{H}$. Incidence of hypoglycemia in newborn infants classified by birthweight and gestational age. Pediatrics 1971;47:831.

9 Sasanow SR, Georgieff $M K$, Pereira GR. Mid-arm circumference and midarm circumference/head circumference ratios: standard curves for anthropometric assessment of neonatal nutritional status. I Pediatr 1986;109: $311-5$

10 Oakley JR, Parsons RJ, Whitelaw AGL. Standards for skinfold thickness in British newborn infants. Arch Dis Child 1977;52:287-90.

11 D'Souza SW, Black P, MacFarlane T, Jennison RF, Richards B. Haematological values in cord blood in relation to fetal hypoxia. Brf Obstet Gynaecol 1981;88:129-32.

12 Beattie RB, Dornan JC, Thompson W. Intrauterine growth retardation: prediction of perinatal distress by Doppler ultrasound. Lancet 1987;ii:974-5.

3 Stuart B, Diumm J, Fitzgerald D, Duignan NM. Fetal blood velocity waveforms in normal pregnancy. Br 7 Obstet Gynaecol 1980 -87:780-5.

4 Lockwood CJ, Weiner S. Assessment of fetal growth. Clin Perinatol 1986;13: $3-35$

15 Fleischer A, Schulman H, Farmakides G, et al. Umbilical artery velocity waveform and intrauterine growth retardation. Am $\mathcal{J}$ Obstet Gynecol waveform and $1985 ; 151: 502-5$.

16 Buchan BC. Maternal and fetal blood viscosity throughout normal pregnancy. Fournal of Obstetrics and Gynaecology 1984:4:143-50.

17 Foley ME, Collins R, MacDonald D. Packed cell volume and whole blood viscosity in the acidotic ferus. Fournal of Obstetrics and Gynaecologv 1983;3:170-2

18 Mentzer W'C. Polycythaemia and the hyperviscosity syndrome in newborn infants. Clinics in Haematology 1978:7:63-7.

19 Joupila P, Kirkinen P. Umbilical venous blood flow as an indicator of fetal hypoxia. Br fobstet (ivnaecol 1984;91:107-10.

20 Sykes GS, Johnson P. Ashworth F, et al. Do Apgar scores indicate asphyxia? Lancet 1982:1:494-6.

21 Dijxhoorn MJ, Visser GH, Fidler VJ, Touwen BC, Huisjes HJ. Apgar score, meconium and acidaemia at hirth in relation to neonatal neurological morbidity in term infants. Br f Obstet Gynecol 1986;93:217-22.

22 Meizner I, Katz. M, Lunenfeld E, Insler V. Umbilical and uterine flow velocity waveforms in pregnancies complicated by major fetal anomalies. Prenat Diagn 1987:7:491-6.

23 Trudinger $\mathrm{BJ}$, Cook CM. Umbilical and uterine arterv flow velocity waveforms in pregnancr associated with major fetal abnormality. $\mathrm{Br} f$ Obstet (ivnuecol in pregnancy as $1985 \cdot 92: 666-70$.

24 Rochelson B, Schulman H, Farmakides $G$, et al. The significance of absent end-diastolic velocity in umbilical artery velocity waveforms. Am $\mathcal{Y}$ Obstet Gynecol 1987:156:1213-8

25 Reed KL, Anderson CF, Shenker L. Changes in intracardiac Doppler blood flow velocities in fetuses with ahsent umbilical artery diastolic flow. Am $\mathcal{f}$ Obstet Gynecol 1987;157:774-9.

26 Neilson JP. Doppler ultrasound. Br F Obslet Gynaecol 1987:94:929-34

Accepled 23 December 1988 